\title{
Implementación de la CT-04 en la asignatura Diseño y Aplicación de Equipos Industriales del Máster Universitario de Ingeniería Industrial
}

\author{
Juan F. Dolsa, Francisco J. Rubio ${ }^{\text {b }}$, Enrique Nadale, Eva M. Sánchez-Orgaz ${ }^{\mathrm{d}}$, Juan \\ Giner-Navarro $^{e}$ y Andrés Rovira ${ }^{\mathrm{f}}$ \\ aInstituto de Diseño y Fabricación (IDF), Universitat Politècnica de València (jdols@mcm.upv.es),

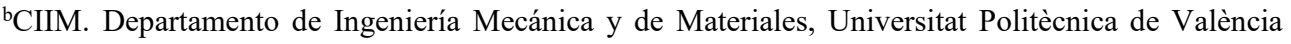 \\ (frubio@mcm.upv.es), ${ }^{c}$ CIIM. Departamento de Ingeniería Mecánica y de Materiales, Universitat \\ Politècnica de València (ennaso@upvnet.upv.es), ${ }^{\mathrm{d} C I I M}$. Departamento de Ingeniería Mecánica y de \\ Materiales, Universitat Politècnica de València (evsncor@upvnet.upv.es), ${ }^{\mathrm{e}} \mathrm{CIIM}$. Departamento de \\ Ingeniería Mecánica y de Materiales, Universitat Politècnica de València (juanginer@upv.es), ${ }^{\mathrm{f}} \mathrm{CIIM}$. \\ Departamento de Ingeniería Mecánica y de Materiales, Universitat Politècnica de València \\ (arovira@mcm.upv.es).
}

\begin{abstract}
This article describes the application of the SCAMPER technique for the evaluation of the CT-04 "Innovation, Creativity and Entrepreneurship", in the subject "Design and Application of Industrial Equipment (DAEI), compulsory of 7.5 ECTS, taught in the Master's Degree in Industrial Engineering (MUII) of the ETSII of Valencia. The analysis presented corresponds to the results of 113 students (36\% of those enrolled), who attended it in the period 2017-18. To evaluate the CT-04 two different activities were proposed: a laboratory practice developed in the 1st partial, and an individual contribution of each student in the final project-work of the subject. The results showed that the type of activities that facilitate the evaluation of the CT-04 should contribute to the final grade of the subject, and have to correspond with the analysis of processes, systems, products or methodologies based on real activities, rather than on designs or products defined theoretically. Therefore, in our case it is recommended that the CT-04 be evaluated exclusively the final work of the subject, done on a company where students can check in-situ systems, methodologies, modes of transport and maintenance, which have to be optimized or innovated later.
\end{abstract}

Keywords: competence, innovation, creativity, entrepreneurship, master

\section{Resumen}

El presente artículo describe la aplicación de la técnica SCAMPER para la evaluación de la CT-04 "Innovación, Creatividad y Emprendimiento", en la asignatura "Diseño y Aplicación de Equipos Industriales (DAEI), troncal y obligatoria de 7,5 ECTS, impartida en el Máster Universitario de Ingeniería Industrial (MUII) de la ETSII de Valencia. El análisis presentado corresponde a los resultados de 113 alumnos (36\% de los matriculados), que la cursaron en el periodo 2017-18. Para evaluar la CT-04 se propusieron dos actividades distintas: una práctica de laboratorio, y una aportación individual de cada 
alumno en el trabajo-proyecto final de la asignatura. Los resultados demostraron que el tipo de actividades que facilita la evaluación de la CT-04 deben contribuir a la nota final de la asignatura, y han de corresponderse con el análisis de procesos, sistemas, productos o metodologías basadas en actividades reales, más que en diseños o productos definidos teóricamente. Por ello, en nuestro caso se recomienda que la CT-04 se evalúe exclusivamente el trabajo final de la asignatura, realizado sobre una empresa donde los alumnos pueden comprobar in-situ los sistemas, metodologias, modos de transporte y manutención, que tienen que optimizar o innovar posteriormente.

Palabras clave: competencia, innovación, creatividad, emprendimiento, máster

\section{Introducción}

Desde la implantación de los nuevos planes de estudio en el marco del Espacio Europeo de Educación Superior (EEES), la mayoría de las universidades españolas han modificado sus curriculums educativos para abordar la consecución de diferentes competencias, agrupadas en específicas, genéricas o transversales, además de impartir los contenidos científicos correspondientes a las diferentes áreas de conocimiento. Este hecho, ha motivado que en la Universitat Politècnica de València (UPV), de acuerdo con su plan estratégico UPV2020 (UPV, 2015a), y como consecuencia de los procedimientos de acreditación realizados para algunas de sus titulaciones por la agencia ABET en 2012, se hayan adoptado hasta 13 conceptos, agrupados en diferentes dimensiones, para acreditar las competencias transversales, y desplegados para evaluar los resultados de aprendizaje en los niveles de dominio asociados al Grado (nivel I y II) y el Máster (nivel III) (UPV, 2015b).

Las Competencias Transversales implementadas por la UPV se han caracterizado por ser integradoras, transferibles, inter-dependientes, multifuncionales y evaluables, estando orientadas a la consecución por parte de los alumnos egresados, de una serie de habilidades cognitivas y metacognitivas, de conocimientos instrumentales y de actitud ante los retos profesionales de la sociedad del conocimiento (UPV, 2015b).

En el presente artículo se va a describir la implementación de la Competencia Transversal CT-04 "Innovación, creatividad y emprendimiento" en una asignatura del Nivel III, Máster Universitario habilitante. Según la UPV, el desarrollo de la CT-04 implica la asunción de una serie de conceptos básicos definidos según estas tres dimensiones. De acuerdo con este planteamiento, la innovación se entiende como "la capacidad de dar respuesta satisfactoria a las necesidades personales, organizativas y sociales, modificando procesos y/o resultados para generar nuevo valor" (UPV, 2015b). Según este concepto, y como ya indicaba el profesor Justo Nieto, "la innovación se materializa en un proceso que intenta conseguir un fin mejor a través del uso del conocimiento" (Nieto, 2008). Por ello, para desarrollar esta competencia, el alumno debe ser capaz de "pensar de otro modo para aportar distintas perspectivas (creatividad), y comprometer determinados recursos por iniciativa propia, con el fin de explorar una oportunidad, asumiendo el riesgo que esto comporta (emprendimiento)" (UPV, 2015b). 
La evaluación de la CT-04 presentada aquí, se llevará a cabo en el Máster Universitario de Ingeniería Industrial (MUII) de la Escuela Técnica Superior de Ingenieros Industriales de Valencia (ETSII), en la asignatura "Diseño y Aplicación de Equipos Industriales (DAEI)", obligatoria y troncal, e impartida en el $2^{\circ}$ cuatrimestre del $1^{\circ}$ curso del MUII. Dicha asignatura tiene un peso específico de 7.5 ECTS (4.0 de teoría y 3.5 de prácticas), siendo la más importante, en peso específico, de las impartidas en la materia "48-Construcciones Industriales e Ingeniería Mecánica", donde comparte troncalidad con otras dos asignaturas: "Construcción, Arquitectura y Urbanismo Industrial" (5.25 ECTS) y "Tecnología de Fabricación" (4.5 ECTS). En el presente artículo se analizará la implementación y evaluación de la CT-04 durante el curso 2017-18, en el que se matricularon un total de 314 alumnos.

Según la normativa y directrices internas establecidas por la UPV para la evaluación de la CT-04 (UPV, 2015b), para un Nivel de Dominio III (Máster), los resultados de aprendizaje deberán estar basados en la propuesta de un plan de acción, para lo cual el alumno debería realizar un análisis global de la innovación propuesta en el problema planteado, y realizar una medición de los avances propuestos en base a la medición de una serie de indicadores, que contemplen;

- La integración de conocimientos de otras disciplinas.

- La adopción de enfoques creativos en el contenido y modo de realización.

- La propuesta de un plan de acción.

- El análisis del valor de la innovación.

Por todo ello, se decidió que para la evaluación de la CT-04 en la asignatura DAEI, se utilizara UNA única técnica, el SCAMPER, pero desarrollada en DOS actividades distintas, a saber:

- en una de las prácticas de laboratorio, concretamente la correspondiente a la práctica $\mathrm{n}^{\mathrm{O}} 3$ sobre sistemas de transporte continuo de cargas horizontales,

- en la aportación individual que realiza cada alumno a la nota final del trabajoproyecto final de la asignatura.

La palabra SCAMPER es un acrónimo de las palabras/términos que definen cada una de sus letras (Eberle, 2008), y que identifican las siguientes ideas:

- $\quad$ SUSTITUIR (SUBSTITUTE)

- combinar (COMBINE)

- aDAPTAR (ADAPT)

- mODIFICAR (MODIFY)

- PONERLO EN OtROS USOS (PUT TO OTHER USES)

- $\quad$ ELIMINAR (ELIMINATE)

- REORDENAR (REARRANGE) 
Para aplicar esta técnica se debe inicialmente identificar el elemento que se desea mejorar. Este elemento podrá ser un producto, un servicio, o también un proceso. En nuestro caso, a la hora de aplicarlo se pensará tanto en el sistema de transporte continuo a implementar en la práctica $\mathrm{n}^{\circ} 3$, como en el análisis del problema de transporte en una empresa real que se debe realizaren el trabajo-proyecto final de la asignatura. A continuación, se deben formular una serie de preguntas utilizando cada una de las acciones anteriores (una cada vez), aplicando éstas al objetivo y tomando nota de las ideas que surgen para ir desarrollándolas a continuación. Se deben revisar los cambios sugeridos, y determinar cuales de éstos se adaptan a los criterios de la solución buscada.

A modo de ejemplo, se muestran a continuación, ejemplos de las preguntas correspondientes a cada verbo/acción del método SCAMPER, aplicado a las actividades evaluadas en la asignatura DAEI ( $3^{a}$ práctica y trabajo final):

\section{SUSTITUIR (SUBSTITUTE)}

- ¿Que no se puede sustituir/cambiar del proceso de transporte analizado?

- ¿Que se puede sustituir del proceso de transporte analizado para bajar costos?

- ¿Qué más puede ser sustituido?

- ¿Se pueden cambiar las reglas?

- ¿Se puede cambiarlo por otro?

- ¿Otros materiales?

- ¿Otra forma (geométrica)?

- ¿Otro proceso o procedimiento?

- ¿Otras fuerzas?

- ¿Otro lugar?, ¿Otro orden?, ¿Otra secuencia?

- ¿Una aproximación diferente?

- ¿Qué se puede sustituir del proceso de transporte para eliminar complejidad del sistema?, etc.

\section{COMBINAR (COMBINE)}

- ¿Qué se puede combinar internamente del proceso de transporte analizado?

- ¿Qué puedes combinar con un factor externo?

- ¿Qué combinación de elementos y/o sistemas generaría una reducción de costos?

- ¿Qué ideas se pueden combinar?

- ¿Qué otros artículos podrían mezclarse con éste?

- ¿Qué se puede combinar para multiplicar los posibles usos?

- ¿Qué materiales podríamos combinar?, etc.

\section{ADAPTAR (ADAPT)}

- ¿Cómo se puede adaptar el proceso de transporte para agregar otra función?

- ¿Qué se puede adaptar para que esté disponible a una mayor cantidad de personas?

- ¿Que podríamos copiar de otros/de otra parte?

- ¿Que otro proceso de transporte se podría adaptar?

- ¿En qué diferentes contextos se puede incluir el concepto desarrollado?

- ¿Qué ideas de otros campos diferentes se pueden incorporar? 
- ¿Qué otro proyecto, proceso o servicio se parece a este?

- ¿Qué idea te sugiere este parecido?, etc.

\section{MODIFICAR (MODIFY)}

- ¿Qué parte del sistema de transporte puede ser modificada para reducir costos?

- ¿Cómo lo podemos alterar para mejorarlo?

- ¿Qué se puede modificar: todo/una parte/un detalle/función?

- ¿Hay alguna peculiaridad en el proceso: diseño, embalaje, protección?

- ¿Qué puede ser modificado para reducir costos de mantenimiento?

- ¿Qué podemos magnificar, aumentar, ampliar, ensalzar o extender?

- ¿Qué podemos añadir: más tiempo, más resistente, más largo, más grande?

- ¿Qué puede dar más valor añadido?

- ¿Se puede duplicar?, etc.

\section{PONERLO EN OTROS USOS (PUT TO OTHER USES)}

- ¿Qué otros usos puede tener?

- ¿Qué modificar para dar otros usos?

- ¿Otros mercados?

- ¿Otros modelos?, etc.

\section{ELIMINAR (ELIMINATE)}

- ¿Qué función del sistema de transporte continuo puede ser eliminada?

- ¿Qué función no puede ser eliminada?

- ¿Si eliminas un atributo como el tamaño, capacidad, velocidad, etc., el costo disminuye?

- ¿Qué pasaría si fuera más pequeño? ¿Qué se debería de omitir?

- ¿Se puede/debe dividir, reducir, hacer más eficiente?

- ¿Qué hay que no sea necesario?

- ¿Qué nos revelaría un diagrama del proceso?, etc.

\section{REORDENAR (REARRANGE)}

- ¿Intercambiar componentes, partes, funciones, sistemas, ...?

- ¿Un modelo diferente?

- ¿Una distribución física diferente? ¿Otra secuencia? ¿Cambiar el orden?

- ¿Cambiar la velocidad? ¿Frecuencia?

- ¿Cambiar la planificación?, etc.

\section{Objetivos}

En el presente documento se describirá la aplicación de la técnica SCAMPER en las dos actividades propuestas ( $3^{\mathrm{a}}$ práctica, y la aportación individual al trabajo final de la asignatura DAEI), así como los métodos de evaluación seguidos para su control. 
La metodología SCAMPER consiste en la aplicación de un check-list que se utiliza para la creación de nuevas ideas sobre mejoras o nuevos productos/servicios, y que da soporte a brainstormings o reflexiones para no dejarse conceptos que pueden ser interesantes (Higgins \& Associates, 2019; MindTools, 2019). Esta técnica de creatividad fue elaborada por Bob Eberle (Eberle, 2008), a partir de una lista de verificación verbal diseñada por Alex Oxborn (Osborn, 1953), el creador del brainstorming, pionero del desarrollo de las técnicas de creatividad. Dichas técnicas de creatividad han sido implementadas posteriormente en numerosos entornos, tanto a nivel empresarial (De la Torre, Hernández y Velaz, 2008; Joachin, 2019), como individual (Schnarch, 2010).

Para realizar una correcta evaluación de la implementación de la CT-04 en la asignatura DAEI del MUII mediante la técnica del SCAMPER, se han planteado en el presente artículo diferentes hipótesis de trabajo. Del análisis de la implementación de las rúbricas desarrolladas por la UPV para su evaluación (Cuenca et al., 2015), y del grado de implantación y aplicabilidad de esta técnica en un nivel de dominio III, correspondiente a un Máster habilitante (UPV, 2015b), se podrán extraer las conclusiones más adecuadas sobre la idoneidad del método elegido.

Las hipótesis de partida que ayudarán a establecer la idoneidad de la metodología desarrollada, se corresponderán con la respuesta a las siguientes preguntas motrices:

i. ¿Se ha evaluado correctamente la CT-04 con cada una de las actividades realizadas?

ii. ¿Es necesario evaluar la CT-04 con las dos actividades propuestas (Trabajo Final y práctica $\left.3^{\mathrm{a}}\right)$ ?

iii. ¿Las notas del Trabajo Final y de la práctica $3^{\mathrm{a}}$ están relacionadas con la nota de la CT-04 evaluada?

\section{Desarrollo de la competencia transversal CT-04 en la asignatura DAEI mediante técnicas SCAMPER}

\subsection{Evaluación de la técnica del SCAMPER en la $3^{\text {a }}$ práctica de laboratorio de DAEI}

En la práctica de aula $n^{\circ} 3$ de la asignatura DAEI (MUII-ETSII), se pretende diseñar conceptualmente un proceso de transporte continuo y automatizado, mediante la implementación de las líneas de trabajo que deben permitir el desplazamiento continuo de diferentes tipos de productos, según las especificaciones de la Tabla 1, y que han de seguir los procesos descritos en el diagrama de flujo definido en la Fig. 1. En dicho proceso, se han de concretar, tanto los sistemas de control por pesos y dimensiones utilizados en las operaciones de transporte, como la concreción y justificación de las características técnicas de todas y cada una de las tipologías de sistemas de transporte continuo, utilizados en cada tramo de línea definido.

Además de la resolución de los problemas técnicos de transporte planteados en esta práctica, para la evaluación de la CT-04 en esta actividad, se propone que cada alumno, a título individual, realice las siguientes actividades: 
1) Aportar nuevas ideas y planteamientos que permitan resolver el problema de transporte y manutención de las actividades industriales que se presentan en la práctica, desde la perspectiva individual del alumno.

2) Analizar los resultados obtenidos en el trabajo del grupo y establecer las conclusiones adecuadas sobre los mismos.

Tabla 1. Especificaciones de las cargas a transportar en el sistema de transporte continuo.

\begin{tabular}{|l|c|c|c|c|}
\hline \multicolumn{1}{|c|}{ Productos Tipo } & Longitud (cm) & Anchura (cm) & Altura (cm) & Peso (kg) \\
\hline 1 - Palets & 100 & 120 & 100 & $100-500$ \\
2 - Bidones & $50(\varnothing)$ & -- & 80 & 50 \\
3 - Cajas & 50 & 50 & 40 & 35 \\
4 - Cajas & 125 & 40 & 25 & 45 \\
5 - Cargas variadas & $10-20$ & $10-25$ & $15-30$ & $5-20$ \\
\hline
\end{tabular}

Esta práctica tiene un peso específico del $3.3 \%$ en la nota final de la asignatura. Pero la realización de los ejercicios planteados para evaluar la CT-04 con la técnica SCAMPER, no contribuyen de ningún modo a la nota final de la asignatura, más allá de facilitar la evaluación de la CT-04 individual, por lo que su realización es meramente voluntaria.

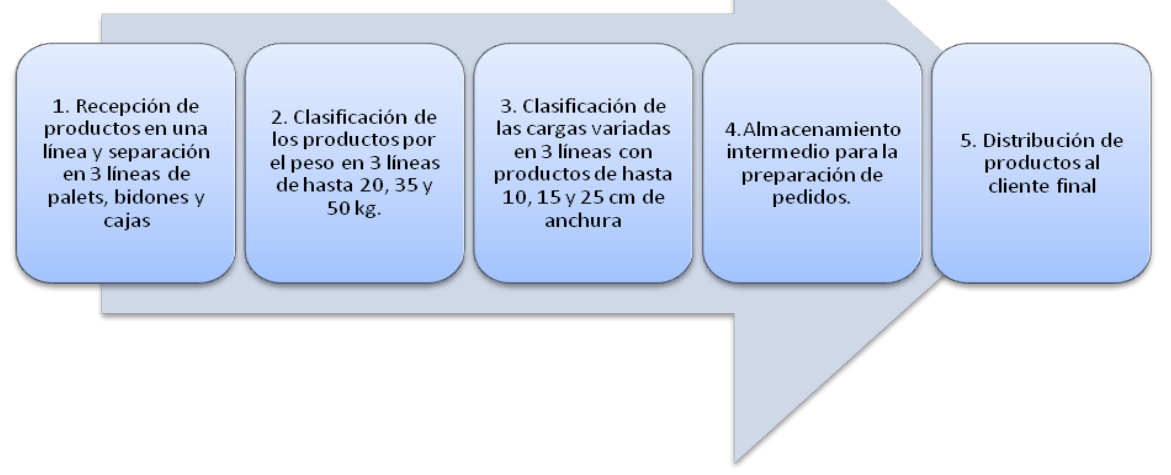

Fig. 1. Diagrama de flujo de las operaciones de manutención continuo a diseñar en la práctica de laboratorio.

La evaluación de esta actividad por parte de los profesores de prácticas de la asignatura, se llevará a cabo mediante la aplicación de la rúbrica que se muestra en la Tabla 2.

Cada profesor debe analizar las respuestas del alumno según las preguntas planteadas en los indicadores de la Tabla 2, y en función del problema propuesto en la práctica 3. Mediante estos indicadores, y ante las soluciones individuales propuestas por cada alumno, se puntua la CT-04 según el criterio definido en los descriptores, asignándole una puntuación numérica entre 0 y 10.

Tabla 2. Rúbrica para la evaluación de la contribución individual mediante la técnica SCAMPER en la práctica de laboratorio 3 . 


\begin{tabular}{|c|c|c|c|c|c|c|}
\hline & $\begin{array}{c}\text { D. No } \\
\text { alcanzado }\end{array}$ & $\begin{array}{c}\text { C. En } \\
\text { desarrollo }\end{array}$ & $\begin{array}{c}\text { B. Bien / } \\
\text { adecuado }\end{array}$ & $\begin{array}{l}\text { A. Excelente / } \\
\text { ejemplar }\end{array}$ & $\begin{array}{l}\text { Ejemplos de } \\
\text { evidencias }\end{array}$ & $\begin{array}{c}\text { Resultado } \\
\text { de la } \\
\text { evaluación } \\
\text { de la CT-04 } \\
\text { en la } \\
\text { práctica } \\
\text { aula } 3\end{array}$ \\
\hline $\begin{array}{c}\text { Adapta enfoques } \\
\text { creativos, } \\
\text { nuevas ideas y } \\
\text { planteamientos } \\
\text { en el desarrollo } \\
\text { de la práctica }\end{array}$ & $\begin{array}{c}\text { No propone } \\
\text { ideas } \\
\text { originales, } \\
\text { ni distintas, } \\
\text { a las } \\
\text { propuestas } \\
\text { por el grupo } \\
\text { en el } \\
\text { desarrollo } \\
\text { de la } \\
\text { práctica }\end{array}$ & $\begin{array}{c}\text { Propone ideas } \\
\text { y/o enfoques que } \\
\text { no se adaptan a } \\
\text { la situación } \\
\text { planteada en la } \\
\text { práctica, y/o no } \\
\text { especifican } \\
\text { correctamente el } \\
\text { modo de } \\
\text { ejecución }\end{array}$ & \begin{tabular}{|} 
Adopta enfoques \\
adecuados al \\
contenido de la \\
práctica, y realiza \\
un correcto \\
planteamiento \\
del modo de \\
desarrollar su \\
aportación
\end{tabular} & $\begin{array}{c}\text { Adopta enfoques } \\
\text { originales, } \\
\text { generando nuevas } \\
\text { ideas que difieren } \\
\text { de las planteadas } \\
\text { por el grupo, y } \\
\text { aporta creatividad } \\
\text { en su propuesta, } \\
\text { mejorando } \\
\text { sistemas, } \\
\text { procedimientos } \\
\text { y/o procesos de } \\
\text { transporte }\end{array}$ & $\begin{array}{c}\text { Aborda la } \\
\text { situación } \\
\text { propuesta por el } \\
\text { grupo como } \\
\text { solución al } \\
\text { problema } \\
\text { planteado, y desde } \\
\text { enfoques } \\
\text { originales, } \\
\text { alternativos a los } \\
\text { del grupo, } \\
\text { propone nuevas } \\
\text { ideas y } \\
\text { procedimientos } \\
\text { originales, que } \\
\text { tienen sentido y se } \\
\text { pueden } \\
\text { materializar como } \\
\text { una mejora } \\
\text { evidente }\end{array}$ & $0 \ldots 10$ ptos. \\
\hline $\begin{array}{c}\text { Analiza el valor } \\
\text { de la innovación } \\
\text { propuesto por el } \\
\text { grupo en el } \\
\text { desarrollo de la } \\
\text { práctica, y } \\
\text { establece } \\
\text { conclusiones }\end{array}$ & $\begin{array}{l}\text { No lleva a } \\
\text { cabo ningún } \\
\text { análisis }\end{array}$ & $\begin{array}{c}\text { Realiza algún } \\
\text { análisis, } \\
\text { identificando } \\
\text { algunas ventajas } \\
\text { e inconvenientes } \\
\text { de los resultados, } \\
\text { de forma } \\
\text { limitada }\end{array}$ & $\begin{array}{l}\text { Realiza un } \\
\text { análisis } \\
\text { adecuado del } \\
\text { valor de la } \\
\text { innovación } \\
\text { planteada por } \\
\text { el grupo en la } \\
\text { práctica }\end{array}$ & $\begin{array}{l}\text { Realiza un análisis } \\
\text { global del valor de } \\
\text { la innovación } \\
\text { planteada por el } \\
\text { grupo, empleando } \\
\text { las herramientas } \\
\text { adecuadas. }\end{array}$ & $\begin{array}{c}\text { Realiza un } \\
\text { informe con el } \\
\text { análisis del valor, } \\
\text { en el que señala } \\
\text { las herramientas } \\
\text { y/o técnicas } \\
\text { utilizadas (p.e. } \\
\text { análisis DAFO, } \\
\text { técnicas multi- } \\
\text { criterio, análisis } \\
\text { de probabilidad, } \\
\text { indicadores de } \\
\text { eficiencia, } \\
\text { económicos, de } \\
\text { calidad, impacto } \\
\text { ambiental, etc.) }\end{array}$ & $0 \ldots 10$ ptos. \\
\hline \multicolumn{6}{|c|}{ Resultado de la evaluación de la CT-04 en la aplicación del SCAMPER en la práctica de aula $\mathrm{n}^{\circ} 3^{(1)}$} & $\mathrm{A}, \mathrm{B}, \mathrm{C}, \mathrm{D}$ \\
\hline
\end{tabular}

(1) El resultado de la evaluación de la CT-04 en esta actividad se calculará obteniendo la media de los dos indicadores considerados en esta rúbrica, y para cada indicador se ponderará una puntuación que se definirá de la siguiente forma:
D. No alcanzado: 0-3.0 puntos.
C. En desarrollo: 3.0-6.0 puntos
B. Bien/adecuado $6.0-8.5$ puntos
A. Excelente/ejemplar: $8.5-10$ puntos

\subsection{Evaluación de la técnica del SCAMPER en el trabajo final.}

En el trabajo final, la evaluación de la CT-04 se llevará a cabo mediante una aportación individual en la entrega del mismo. Dicha aportación pesará un $5 \%$ de la nota final del trabajo, y en la misma, cada miembro del grupo realizará algún tipo de propuesta de mejora en el problema de transporte analizado o en la instalación evaluada.

El trabajo-proyecto final de la asignatura DAEI, se plantea como un trabajo de campo realizado por un grupo de alumnos (2-3) sobre una empresa real, de actividad industrial contrastable. En el trabajo se deben analizar los problemas de procesos, sistemas y/o modos de transporte estudiados en la asignatura y aplicar los conocimientos adquiridos, en base a los que tienen que proponer soluciones que mejoren, optimicen o sustituyan a los sistemas de transporte o manutención analizados. El trabajo tiene un peso específico del $25 \%$ de la nota final de la asignatura, y debe ser defendido en una presentación oral en público. La 
aportación individual mediante la cual se evalúa la CT-04 en esta actividad, tiene un peso específico del $5 \%$ de la nota del trabajo, por lo que el porcentaje de esta contribución sobre la nota final de la asignatura es de sólo el 1,25\%.

En la rúbrica de la aportación individual de cada alumno, se tendrán en cuenta aspectos como la aplicación de la técnica SCAMPER para la generación de la idea de mejora del proyecto que se propone, y que se resume en la siguiente rúbrica de evaluación (Tabla 3):

Tabla 3. Rúbrica para la evaluación de la contribución individual mediante la técnica SCAMPER en el Trabajo Final de la asignatura DAEI.

\begin{tabular}{|c|c|c|c|c|c|c|}
\hline \multirow[b]{2}{*}{ INDICADORES } & \multicolumn{4}{|c|}{ DESCRIPTORES } & \multirow[b]{2}{*}{$\begin{array}{l}\text { Ejemplos de } \\
\text { evidencias }\end{array}$} & \multirow{2}{*}{$\begin{array}{c}\text { Resultado } \\
\text { evaluación } \\
\text { CT-04 en } \\
\text { la práctica } \\
\text { aula } 3\end{array}$} \\
\hline & $\begin{array}{c}\text { D. No } \\
\text { alcanzado }\end{array}$ & $\begin{array}{c}\text { C. En } \\
\text { desarrollo }\end{array}$ & $\begin{array}{l}\text { B. Bien / } \\
\text { adecuado }\end{array}$ & $\begin{array}{l}\text { A. Excelente } \\
\text { / ejemplar }\end{array}$ & & \\
\hline $\begin{array}{c}\text { Adapta enfoques } \\
\text { creativos, } \\
\text { nuevas ideas y } \\
\text { planteamientos } \\
\text { en el desarrollo } \\
\text { del trabajo final }\end{array}$ & $\begin{array}{c}\text { No propone } \\
\text { ideas } \\
\text { originales, ni } \\
\text { distintas, a las } \\
\text { propuestas por } \\
\text { el grupo en el } \\
\text { desarrollo del } \\
\text { trabajo final }\end{array}$ & $\begin{array}{c}\text { Propone ideas y/o } \\
\text { nfoques que no se } \\
\text { adaptan a la } \\
\text { situación } \\
\text { planteada en el } \\
\text { trabajo final, y/o } \\
\text { no especifican } \\
\text { correctamente el } \\
\text { modo de ejecución }\end{array}$ & \begin{tabular}{|c|} 
Adopta enfoques \\
adecuados al \\
contenido del \\
trabajo final, y \\
realiza un correcto \\
planteamiento del \\
modo de \\
desarrollar su \\
aportación
\end{tabular} & \begin{tabular}{|} 
Adopta enfoques \\
originales, generando \\
nuevas ideas que \\
difieren de las \\
planteadas por el \\
grupo, y aporta \\
creatividad en su \\
propuesta, mejorando \\
sistemas, \\
procedimientos y/o \\
procesos de \\
transporte
\end{tabular} & \begin{tabular}{|c|} 
Aborda la situación \\
propuesta por el grupo \\
como solución al problema \\
planteado, y desde \\
enfoques originales, \\
alternativos a los del \\
grupo, propone nuevas \\
ideas y procedimientos \\
originales, que tienen \\
sentido y se pueden \\
materializar como una \\
mejora evidente
\end{tabular} & $0 \ldots 10$ ptos. \\
\hline $\begin{array}{c}\text { Analiza el valor } \\
\text { de la innovación } \\
\text { propuesto por el } \\
\text { grupo en el } \\
\text { desarrollo del } \\
\text { trabajo final, y } \\
\text { establece } \\
\text { conclusiones }\end{array}$ & $\begin{array}{c}\text { No lleva a } \\
\text { cabo } \\
\text { ningún } \\
\text { análisis }\end{array}$ & \begin{tabular}{|} 
Realiza algún \\
análisis, \\
identificando \\
algunas ventajas e \\
inconvenientes de \\
los resultados, de \\
forma limitada
\end{tabular} & $\begin{array}{c}\text { Realiza un análisis } \\
\text { adecuado del valor } \\
\text { de la innovación } \\
\text { planteada por el } \\
\text { grupo en el trabajo } \\
\text { final }\end{array}$ & \begin{tabular}{|c|} 
Realiza un análisis \\
global del valor de la \\
innovación planteada \\
por el grupo, \\
empleando las \\
herramientas \\
adecuadas.
\end{tabular} & \begin{tabular}{|c} 
Realiza un informe con el \\
análisis del valor, en el \\
que señala las \\
herramientas y/o técnicas \\
utilizadas (p.e. análisis \\
DAFO, técnicas multi- \\
criterio, análisis de \\
probabilidad, indicadores \\
de eficiencia, económicos, \\
de calidad, impacto \\
ambiental, etc.)
\end{tabular} & $0 \ldots 10$ ptos. \\
\hline Resu & & ofater & 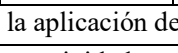 & De & trabajo final $^{(1)}$ & $\mathrm{A}, \mathrm{B}, \mathrm{C}, \mathrm{D}$ \\
\hline
\end{tabular}

(1) El resultado de la evaluación de la CT-04 en esta actividad se calculará obteniendo la media de los dos indicadores considerados en esta rúbrica, y para cada indicador se ponderará una puntuación que se definirá de la siguiente forma:
D. No alcanzado: 0-3.0 puntos.
C. En desarrollo: 3.0-6.0 puntos
B. Bien/adecuado 6.0-8.5 puntos
A. Excelente/ejemplar: 8.5-10 puntos

Cada profesor del grupo de teoría que evalúa el trabajo final, debe analizar las respuestas del alumno, según los indicadores planteados en la Tabla 3, que en función del problema analizado en el trabajo final de la asignatura, son:

- Adapta enfoques creativos, nuevas ideas y planteamientos en el desarrollo del trabajo.

- Analiza el valor de la innovación propuesto por el grupo en el desarrollo del trabajo, y establece conclusiones.

Y ante las soluciones individuales, evalúa según el criterio definido en los descriptores, asignándole una puntuación numérica entre 0 y 10. 


\subsection{Evaluación final de la CT-04 mediante la técnica del SCAMPER en la asignatura DAEI.}

La evaluación final de la CT-04 mediante la aplicación de la técnica SCAMPER en la asignatura DAEI, se obtendrá mediante la implementación de las rúbricas que se muestran en las Tablas 2 y 3 . Las dos puntuaciones numéricas obtenidas en estas rúbricas servirán para obtener una media aritmética, y determinar el resultado de la evaluación de la CT-04 según los valores $\mathrm{A}, \mathrm{B}, \mathrm{C}$ o $\mathrm{D}$.

\section{Resultados}

En primer lugar, a la hora de realizar el análisis de los resultados obtenidos se pudo constatar que, debido al carácter voluntario de la actividad planteada en la práctica $\mathrm{n}^{\mathrm{o}} 3$ para evaluar la CT-04 (que no contaba para la nota final de la asignatura), muchos alumnos no la contestaron. Del mismo modo, como la contribución individual del trabajo final en el que se evaluaba la CT-04, aunque sí contribuía a la nota final, tenía un peso específico muy bajo en la misma $(1,25 \%)$, provocó que muchos alumnos tampoco la presentaran. Por esa razón, de los 314 alumnos matriculados en la asignatura DAEI en el curso 2017-18 del MUII de la ETSII, sólo se pudieron analizar las contribuciones de 113 alumnos (36\%), que sí contribuyeron con los cuatro indicadores evaluables de la CT-04 ( 2 en la práctica ${ }^{\circ} 3$ y 2 en el trabajo final).

El resto de los 201 alumnos (64\%), o no realizaron ninguna contribución para ser evaluados por la CT-04 en ninguna de las actividades planteadas, o sólo lo hicieron en alguna de ellas. En cualquiera de los casos, estos alumnos han sido excluidos del análisis final de la CT-04, para no contaminar los resultados obtenidos y su correlación, que se ha centrado en los 113 alumnos que han contribuido en todas la actividades, y cuyo análisis se presenta a continuación.

\subsection{Resultados de la evaluación de la competencia transversal CT-04}

La Fig. 2 muestra las notas de la CT-04 obtenidas en la práctica 3 y en el trabajo final. La nota para la CT-04 se obtiene como la media aritmética entre estas dos notas y la distribución de notas para los estudiantes se muestra en la Fig. 3.

Se puede observar como la nota de la CT-04 obtenida en la práctica 3 está entre 3 y 9 y, en el trabajo final, está entre 6 y 10, aproximadamente. Estas variaciones se pueden explicar en la propia naturaleza de las actividades planteadas. En la práctica ${ }^{\circ} 3$, el problema a resolver se propone sobre una instalación inexistente que, en teoría, debe ser diseñada conceptualmente por los alumnos, y sobre la cual deben aplicar la técnica SCAMPER para mejorar o innovar la solución obtenida por el grupo. Como esta solución ha sido consensuada por los alumnos para presentar la práctica, resulta más difícil que cada miembro del grupo, en particular, encuentre una alternativa más innovadora que mejore la solución propuesta. Por otro lado, el planteamiento de mejora u optimización de instalaciones industriales de manutención o transporte del trabajo final, se realizará sobre un proceso o sistema ya diseñado, esto es, que esta operativo y en funcionamiento, por lo que entendemos que, a la hora de plantear la metodología del SCAMPER, resulta más sencillo para el alumno innovar o crear algo nuevo sobre lo ya observado en una instalación real. De ahí, que la diferencia de 
notas asignadas a la CT-04 pueda justificarse en base a la existencia real o no de la instalación a analizar.

Otro factor que podría contibuir a esta diferencia en las notas asignadas para cada actividad de la CT-04, es el hecho de que los criterios a aplicar por parte de los profesores que la evalúan, de prácticas por una parte ( $3^{a}$ práctica), y de teoría por otra (trabajo final), pueda generar una diferencia de criterios de evaluación significativa. Esto puede ser debido, no sólo por la dificultad de evaluar las innovaciones propuestas por los alumnos sobre instalaciones que existen sólo en teoría, por un lado, o que ya están en funcionamiento por otro, sino por la falta de experiencia o conocimientos para establecer la aplicación de un criterio objetivo sobre el procedimiento. Lo cual nos indica que, de persistir en esta metodología de evaluación de la CT-04, la asignación de criterios de evaluación debería ser más objetiva y precisa.

Por tanto, al hacer la media entre las dos actividades evaluadas, los valores son relativamente bajos, oscilando alrededor de 7. Así, aplicando la clasificación en la escala categórica de A a $\mathrm{D}$, según las Tablas 2 y 3 , apenas se obtienen estudiantes con nota A y sí muchos estudiantes con notas $\mathrm{B}$ y C. Hay pocos estudiantes que obtengan una calificación de $\mathrm{D}$, resultado que es esperable.
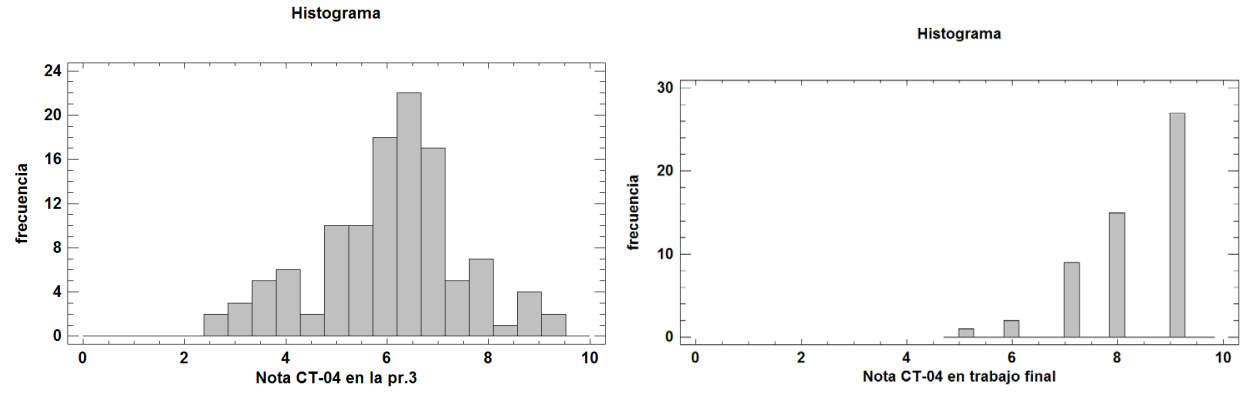

Fig. 2. Notas obtenidas en la CT-04 en la práctica 3 (izquierda) y en el trabajo final (derecha).
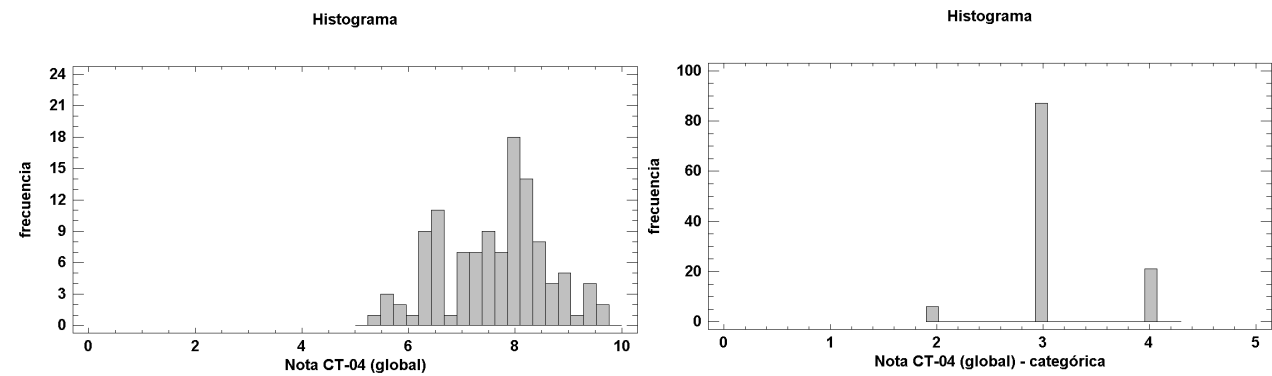

Fig. 3. Notas numéricas de la CT-04 (global) (izquierda) y en escala categórica: A (excelente) = 4, B (bien/adecuado $)=3, C$ (en desarrollo $)=2, D($ No alcanzado $)=1($ derecha $)$.

\subsection{Correlaciones de las notas en las distintas pruebas de evaluación con la nota de la CT-04}

En este punto se presentan algunas correlaciones que se han calculado para comprobar si existe relación entre las notas obtenidas en las distintas pruebas de evaluación y las notas de la CT-04. 
Si se compara la nota de la CT-04 obtenida en la práctica $n^{0} 3$, con la calificación de esta práctica (Fig. 4), se observa que no existe una relación directa entre ambos valores. Este resultado es esperable, ya que por un lado se evalúan los conocimientos, y por otro, la competencia transversal. Mientras la nota de la práctica $n^{\circ} 3$ varía entre 6 y 10, la nota de la CT-04, en esta práctica, varía entre 2 y 10, aproximadamente. Pueden existir algunos casos extremos de evaluaciones en los que la nota de la CT-04 es muy baja (2), y la nota final de la práctica es muy buena (9). En el otro extremo, también se ha observado que en varios casos la nota de la práctica es de las más bajas (6-7), mientras que la evaluación de la CT-04 ha sido buena o muy buena (8-9).

Por otro lado, si comparamos la nota de la CT-04 evaluada en el trabajo final, y la comparamos con la calificación final del trabajo (Fig. 5), observamos que tampoco existe una correlación directa entre ambos valores, aunque en este caso, al contrario de lo que ocurre con la práctica $\mathrm{n}^{\mathrm{0}} 3$, el grado de dispersión de datos es menor. Así, mientras que la nota de la CT-04 es valorada en el trabajo con valores que oscilan entre 5 y 10, la nota del trabajo final varía entre 7 y 10. Así y todo, se observa cómo una buena valoración del trabajo final (nota superior a 8), se corresponde, en términos generales, con valoraciones de la CT-04 por encima de 8. Este hecho puede ser atribuible a, como hemos comentado previamente, la facilidad con la que los alumnos pueden proponer innovaciones sobre instalaciones analizadas que ya existen previamente, en lugar de realizar el proceso creativo sobre diseños teóricos desarrollados en una práctica.

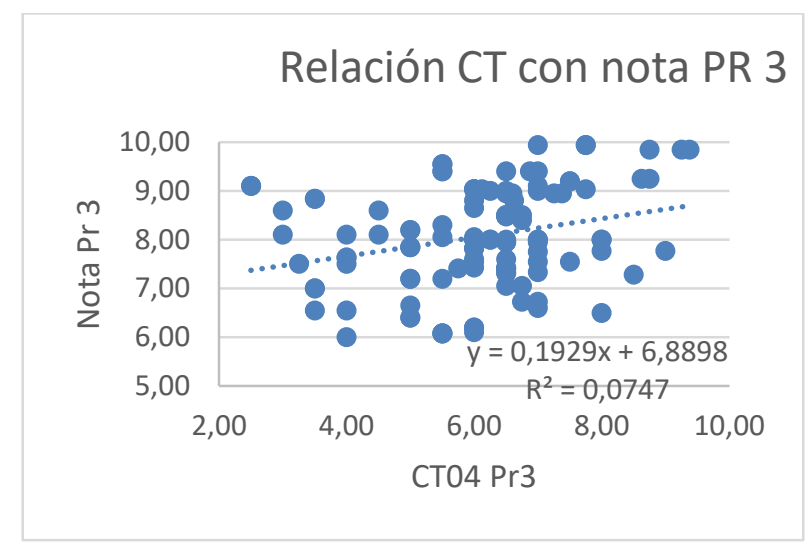

Fig. 4. Relación entre la nota de la CT-04 en la práctica 3 y la calificación de esta práctica. 


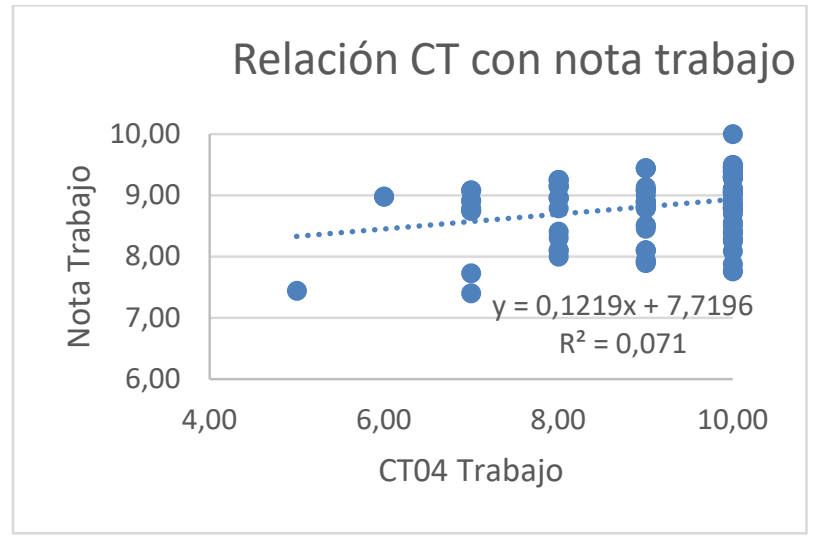

Fig. 5. Relación entre la nota de la CT-04 en la práctica 3 y la calificación de esta práctica.

Finalmente, se compara la relación entre la nota de la CT-04 (global) y la nota final de la asignatura (Fig. 6). Al igual que en los casos anteriores, al realizar la media aritmética de todas las notas que contribuyen a evaluar la CT-04, no existe correlación entre ambos valores, como era de esperar. La nota final de la asignatura oscila entre 5 y 9 mientras la nota de la CT-04 oscila entre 5 y 10, aproximadamente, pero sin observarse correlación.

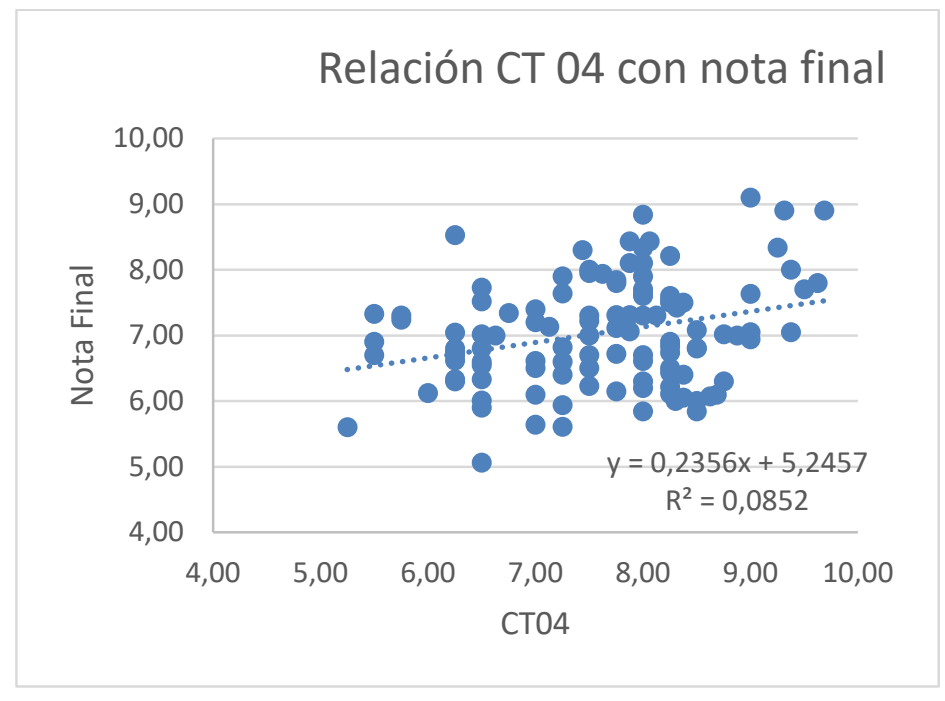

Fig. 6. Relación entre la nota de la CT-04 (global) y la nota final de la asignatura.

\section{Conclusiones}

En el presente artículo se ha descrito la aplicación de la técnica SCAMPER para la evaluación de la Competencia Transversal CT-04 "Innovación, Creatividad y Emprendimiento", en una asignatura troncal y obligatoria del Máster Universitario de Ingeniería Industrial (MUII) de la Escuela Técnica Superior de Ingenieros Industriales de Valencia (ETSII). La asignatura, denominada "Diseño y Aplicación de Equipos Industriales (DAEI)", es impartida en el $2^{\circ}$ cuatrimestre del $1^{\circ}$ curso del MUII. El análisis presentado se corresponde con los resultados analizados de 113 alumnos (36\% de los matriculados), que cursaron la misma durante el periodo 2017-18. La metodología SCAMPER consiste en la aplicación de un check-list, 
creado por Bob Eberle (Eberle, 2008), basado en la creación de nuevas ideas sobre mejoras o nuevos productos/servicios, y que da lugar a brainstormings o reflexiones sobre nuevos conceptos o diseños que pueden ser relevantes para mejorar u optimizar las ideas o soluciones iniciales (Schnarch, 2010; Higgins \& Associates, 2019; MindTools, 2019).

Para realizar una correcta evaluación sobre la implementación de la CT-04 en la asignatura DAEI del MUII mediante la técnica del SCAMPER, se propusieron dos actividades distintas, a saber: una de las prácticas de laboratorio, y una aportación individual de cada alumno en el trabajo final de la asignatura. Del análisis de los resultados obtenidos, y de las hipótesis de trabajo planteadas inicialmente, se obtuvieron las siguientes conclusiones:

- El nivel de respuesta del alumno para la realización voluntaria de las actividades que permiten evaluar la CT-04, depende de si la actividad propuesta contribuye directamente a la nota final de la asignatura. Así, una actividad como la práctica $\mathrm{n}^{\mathrm{o}} 3$, donde la solución propuesta por el alumno para evaluar la CT-04 no contribuye a la nota final, no ha sido realizada por gran parte, casi $2 / 3$, de los alumnos matriculados.

- En actividades donde la evaluación de la CT-04 esta basada en aportaciones individuales del alumno que contribuyen en un valor poco significativo a la nota final, no son realizadas por estos. Es el caso de la aportación individual al trabajo final de la asignatura, cuyo peso específico en la nota final es de sólo un 1,25\%.

- Sólo se debería evaluar la CT-04 mediante la implementación de actividades que, directa o indirectamente, puedan contribuir significativamente a la nota final de la asignatura, evitando así que los alumnos opten por la aplicación de la "ley del mínimo esfuerzo".

- Se debería evitar la evaluación de la CT-04 en actividades diferentes, con diferentes contextos de evaluación y donde participen diferentes tipos de profesores. En ese sentido, los criterios de evaluación de la CT-04 deberían ser claros, establecidos de forma objetiva, y con actividades que puedan ser evaluadas en el mismo contexto docente.

- Es recomendable que el tipo de actividades a realizar para evaluar la CT-04 se corresponda con el análisis de procesos, sistemas, productos o metodologías basadas en casos reales, más que en diseños o productos definidos teóricamente.

- No se ha observado correlación entre la evaluación de la CT-04 y la calificación final de la asignatura, hecho que era esperable ya que los indicadores miden distintos aspectos.

- Se recomienda finalmente que la evaluación de la CT-04 se realice exclusivamente en una actividad como el trabajo final de la asignatura, realizado sobre una empresa con actividad real, donde los alumnos pueden comprobar, in-situ, los procesos, sistemas, metodologías, modos de transporte y manutención, que posteriormente tienen que optimizar o innovar, en base a los hallazgos y análisis realizados en el trabajo. 
- La rúbrica de evaluación de la CT-04 desarrollada para el trabajo final debería ser rediseñada, para que su cumplimentación por parte del profesorado fuera más sencilla y rápida, de modo que los criterios de evaluación quedaran sujetos a menores incertidumbres.

\section{Referencias}

EBERLE, B. (2008). Scamper. Texas, USA: Prufrock Press Inc.

CUENCA, L., ALARCÓN, F., BOZA, A. GERNÁNDEZ-DIEGO, M., RUÍZ, L., GORDO, ML., POLER, R., ALEMANY, M.M.E. (2015). "Rúbrica para la Evaluación de la Competencia Innovación, Creatividad y Emprendimiento en máster". Congreso In-Red 2015-Universitat Politècnica de València. Disponible en http://ocs.editorial.upv.es/index.php/INRED/INRED2015

DE LA TORRE, J. R., HERNÁNDEZ, J., VELAZ, D. (2008). Guía Práctica. La gestión de la Innovación en 8 pasos. Navarra (España). Asociación de la Industria Navarra (AIN).

HIGGINS \& ASSOCIATES. Creatividad e Innovación. Técnicas de creatividad. Scamper. $<$ http://www.innovaforum.com/index2_e.htm>. [Consulta: 21/03/19]. Institucional.

JOACHIN, C.V. UNIVERSIDAD AUTÓNOMA DE MEXICO (UNAM). La creatividad: concepto, técnicas y aplicaciones. Unidad de Apoyo para el aprendizaje (UAPA).

$<\underline{\text { https://programas.cuaed.unam.mx/repositorio/moodle/pluginfile.php/166/mod_resource/c }}$ ontent/1/la-creatividad/index.html> [Consulta: 21/03/19]. Institucional.

Mind Tools - SCAMPER Improving Products and Services. Management Training and Leadership Training, Online. $<$ http://www.mindtools.com/pages/article/newCT_02.htm $>$. [Consulta: 21/03/19]. Institucional.

NIETO, J. (2008). Y tú..., ¿Innovas o Abdicas?. Valencia:Universidad Politécnica de Valencia.

OSBORN, A. (1953). Applied Imagination. New York: hijos de Charles Scribner, 1953.

SCHNARCH, A. (2010). Creatividad aplicada. Cómo estimular y desarrollar la creatividad a nivel personal y empresarial. Starbook editorial, Paracuellos del Jarama, Madrid.

UNIVERSITAT POLITÉCNICA DE VALENCIA. (2015a). Plan Estratégico UPV 20152020. Valencia. $<$ http://www.upv.es/organizacion/lainstitucion/documentos/Plan_Estrategico_UPV2020_int.pdf $>$ (Consulta: 22-03-2019). UNIVERSITAT POLITÉCNICA DE VALENCIA. (2015b). Competencias Transversales $U P V$. Valencia. < www.upv.es/contenidos/COMPTRAN> (Consulta: 21-03-2019). 